\title{
Electrical Conduction and Luminescence for Inverted-Type Organic Light-Emitting Diodes with Polyethyleneimine
}

\author{
Tatsuo Mori*, Satoru Aoyama, and Yoshiyuki Seike \\ Department of Electrical and Electronic Engineering, Graduate School of Engineering, \\ Aichi Institute of Technology, \\ 1247 Yachigusa, Yakusa-cho, Toyota, Aichi 470-0392, Japan \\ *t2mori@aitech.ac.jp
}

\begin{abstract}
Organic light-emitting diodes (OLEDs) have showed the unique potentials of display by bendable, foldable and storable panels. In general, since OLEDs consisted of multi organic layers, their electrical conduction would be complicated. Aluminum quinoline (Alq3) was a classical and famous fluorescent material. We studied the conduction properties of inverted Alq3-bilayer OLEDs. Since the barrier height of electron injection from ITO cathode to Alq3 was higher than that from LiF/Al, electrons were little injected into Alq3 and the current density in the absence of EL was controlled by holes. In the specimen using polyethyleneimine as an injection layer, the current density and hole injection were influenced by electron injection for the current continuity. Despite that holes could arrive at the counter cathode through Alq3, the recombination zone was located near a hole transport layer as the conventional bilayer OLED. It was suggested that the carrier transport in an organic layer depends on both carrier injections.
\end{abstract}

Keywords: Organic light-emitting diode, Inverted structure, Polyethyleneimine, Carrier injection

\section{Introduction}

Organic light-emitting diodes (OLEDs) are used as the display of smartphones and tablets except for liquid crystal displays (LCDs). The bendable [1] and foldable [2] OLED displays have been also developed recently. These displays are expected as the unique solution not available on LCDs. In addition, the panels manufacture by LG Electronics are supplied as a wide TV. And LG Electronics developed a storable TV by winding up [3]. Therefore, it may be felt that the electrical conduction mechanism in organic multi-layer thin films would be clarified physically since the OLEDs consisted of organic multi-layer thin films. However, the electrical conduction will change sensitively by a small difference of thickness and the energy step at some interface, etc. For example, the electrical conduction of $N, N^{\prime}$-di(1-naphthyl) - $N, N^{\prime}$-diphenyl1,1 '-diphenyl-1,4'-diamine) ( $\alpha$-NPD), which is a typical hole-transport material of OLEDs, can describe a simple space charge-limited current (SCLC) if the thickness of $\alpha$-NPD is less than 50 $\mathrm{nm}$ and hole injection is controlled by an appropriate Ohmic contact [4]. But when the thickness is over $50 \mathrm{~nm}$, i.e. $100 \mathrm{~nm}$, the electrical conduction of $\alpha$-NPD layer changes to the PoolFrenkel type field-dependent SCLC [5]. On the other hand, aluminum quinoline (Alq3) is a famous and classical material as an electron transport material and an emitting material [6]. The electrical conduction of Alq3 layer is reported by many researchers [7-9]. However, their current density voltage curves do not conform unfortunately. Consequently the universal and detailed electrical conduction of Alq3 layer is not determined until now, although some commercial device simulators for organic device come onto the market. Therefore, there is room to consider the electrical conduction of organic multi-layer thin films.

In the device structure of conventional OLEDs [6], organic and inorganic layers are sequentially deposited from the anode of oxide semiconductor to a metal cathode on a substrate. The inverted structure whose organic and inorganic layers are sequentially deposited from the cathode to semitransparent inorganic anode is also known $[10,11]$. 
Especially Morii et al. reported the inverted OLED using a semi-transparent metal oxide as an anode [12]. They called this dye-sensitized solar cell (DSC)-based OLED a hybrid organic-inorganic LED (HOILED). The metal with a low work function is widely used as the cathode in the conventional OLED. Since it is oxidized easily, the oxidation of the cathode is one of factors on the device degradation. However, the use of metal oxide cathode and appropriate electron injection material realized the high performance and long lifetime OLED [13].

In general, the work function of metal oxide, i.e. indium-tin-oxide (ITO) and $\mathrm{ZnO}$, is higher than 5.0 $\mathrm{eV}$. Some electron injection materials must be used: AlN [10,11], $\mathrm{TiO}_{2}$ [12], and AZO [13]. Recently polyethyleneimine (PEI) [14] and ethoxylated polyethyleneimine (PEIE) [15] including polar amine groups are famous.

In this paper, we use Alq3 as an emitting and electron transport layers. Since the LUMO of Alq3 is lower than those of poly(9,9-dioctylfluorene-altbenzothiadiazole) (F8BT) [16] and super yellow [17], Alq3 has no aptitude for the inverted OLED. However, we report the EL properties of Alq3-based inverted OLED since they show some interesting results.

\section{Experimental}

\subsection{Materials and fabrication}

We used $\alpha$-NPD as a hole-transport material and 8-hydroxyquinoline aluminum (Alq3) as an emission material from Nippon Steel Chemical Co. The substrates were cleaned by acetone, pure water and 2-propanol with ultrasonic cleaning method. Finally they were cleaned by UV-ozone cleaning. Polyethyleneimine (PEI) (sigma-aldrich) was dispersed $0.4 \mathrm{wt} \%$ in ethanol. Cleaned ITO substrates were immersed in PEI solution. After they were annealed, they were washed by pure water and dried again. Organic thin films were prepared on substrates. The organic thin films were prepared using the vacuum deposition at a pressure of $0.8 \sim 1 \times 10^{-3} \mathrm{~Pa}$ at room temperature. We carried out a vacuum-deposition with EO-55 (EIKO Co.). The conventional structure was ITO/ $\alpha$-NPD (50 $\mathrm{nm}) / \operatorname{Alq} 3(50 \mathrm{~nm}) / \mathrm{LiF}(0.8 \mathrm{~nm}) / \mathrm{Al}$. The inverted structure was ITO/PEI/Alq3 $(50 \mathrm{~nm}) / \alpha-\mathrm{NPD}(50$ $\mathrm{nm}) / \mathrm{MoO}_{3}(0.8 \mathrm{~nm}) / \mathrm{Ag}(30 \mathrm{~nm})$.

\subsection{Measurements}

The work functions of ITO and organic materials are measured with the photoemission yield spectroscopy in air (AC-2, Riken Keiki Co.). Thickness was measured with a surface tester (Kosaka Laboratory, Surfcorder, ET200). Luminance-current-voltage characteristics were measured using a source measure unit (Keithley 2400 Source Measure Unit) and a luminance meter (TOPCON BM-8). The measurements of luminance-current-voltage characteristics were carried out under vacuum of $\sim 0.1 \mathrm{~Pa}$.

\section{Results and discussion}

\subsection{Work function of ITO and ITO/PEI}

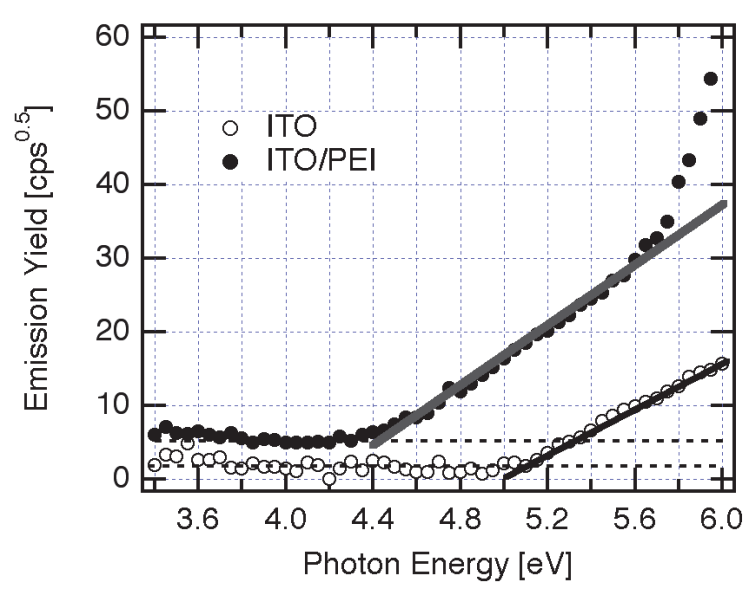

Fig. 1. The emission yield vs. photon energy of bare ITO and PEI-modified ITO.

Figure 1 shows the emission yield vs. photon energy of bare ITO and PEI-modified ITO. The work functions of bare ITO and PEI-modified ITO are estimated to be 5.1 and $4.4 \mathrm{eV}$, respectively. The work function of electrode lowers $0.7 \mathrm{eV}$ by the modification of PEI. The work function of fluorinated self-assembled monolayer (FSAM) modified ITO is $\sim 5.5 \mathrm{eV} \mathrm{[18]} \mathrm{and} \mathrm{is} \mathrm{higher} \mathrm{than} \mathrm{the}$ above values. In the FSAM-modified ITO, the dipole moments of FSAM molecules are facing down to the ITO substrate since each FSAM molecule is adsorbed to ITO via silanol group. The vacuum level of FSAM/ $\alpha$-NPD is shifted to the upper side by the electric double layer. Consequently the energy gap between the work function of ITO and the ionization potential of $\alpha$ NPD becomes smaller and then holes are easily injected from ITO to $\alpha$-NPD. On the other hand, since nitrogen atoms of amine groups are adsorbed to ITO in the PEI-modified ITO, the dipole moments of amine groups of PEI are facing up to the ITO substrate. The vacuum level of PEI/Alq3 is shifted to the down side by the electric double layer with the inverse direction. Consequently, since the electron affinity of PEI/Alq3 will be also shifted to 
the down side, the energy gap between the work function of ITO and the ionization potential of Alq3 will become smaller.

\subsection{Electrical conduction and EL properties}

Figure 2 shows the current density - luminance -voltage (upper) and luminance - current density (lower) characteristics for ITO/PEI or none/ Alq3 $(50 \mathrm{~nm}) / \alpha-\mathrm{NPD}(50 \mathrm{~nm}) / \mathrm{MoO}_{3} / \mathrm{Ag}$. Unfortunately some our inverted-type specimens show instable properties, especially in a low voltage region. The current density of the specimen with PEI is lower than that of the specimen without PEI in spite of low barrier height of electron injection due to lowering work function.
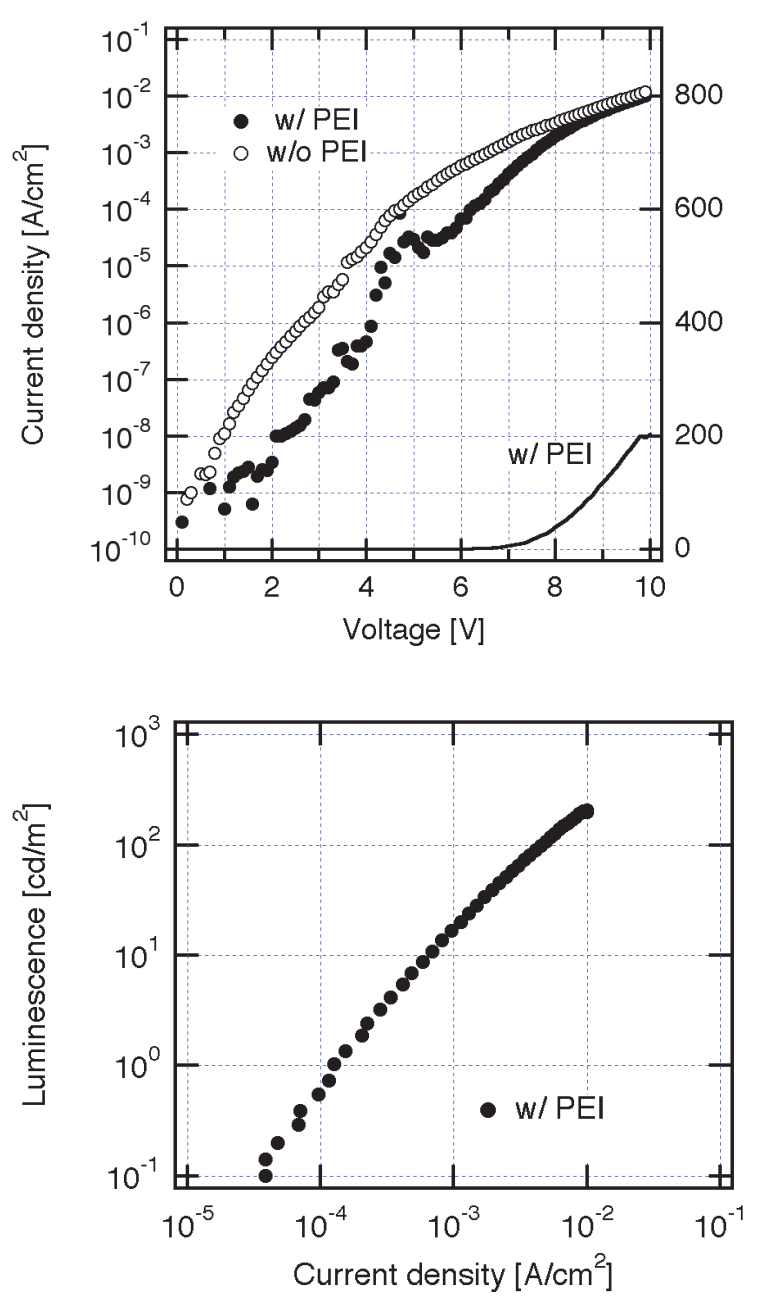

Fig. 2. The current density (circles) - luminance (line) voltage (upper) and luminance - current density (lower) characteristics for ITO/PEI or none/Alq3 (50 nm)/ $\alpha$ $\mathrm{NPD}(50 \mathrm{~nm}) / \mathrm{MoO}_{3} / \mathrm{Ag}$.

The EL is not observed for the specimen without PEI. This means that electrons are not injected from the bare ITO cathode and carrier recombination does not occur in Alq3 layer. On the other hand, the EL starts from $\sim 7 \mathrm{~V}$ for the specimen with PEI. Although the current density for the specimen with PEI is instable, the unstable behavior disappears with increasing electron injection. The current density for the specimen with PEI approaches to that for the specimen without PEI and both show almost same value.

Figure 3 shows the energy band diagram of the specimens with and without PEI. The barrier height of electron injection for the specimen without or with PEI is estimated to be $2 \mathrm{eV}$ or $1.3 \mathrm{eV}$, respectively. Since the electron affinity of F8BT is reported to be $3.5 \mathrm{eV}$ [16], the barrier height of electron injection is estimated to be $0.9 \mathrm{eV}$ and is a little lower than that of Alq3-based OLED.

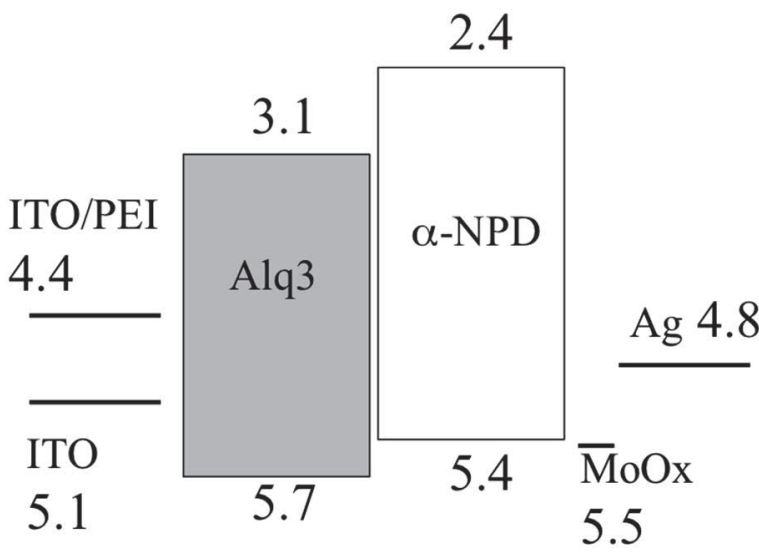

Fig. 3. The energy band diagram of ITO (cathode)/PEI or none/Alq3 $(50 \mathrm{~nm}) / \alpha-\mathrm{NPD}(50 \mathrm{~nm}) / \mathrm{MoO}_{3} / \mathrm{Ag}$ (anode): unit, eV.

When we discuss the carrier injection from an electrode, we often pay much attention for only energy level at the interface as mentioned above. In the steady state, however, one carrier injection is influenced by the counter carrier injection because of the current continuity law. The current density for the specimen without PEI will be controlled by holes injected from the anode. In the specimen without PEI, no electrons will be injected from the ITO cathode because of high barrier height of electron injection. The hole injection from the anode will not be influenced by electron injection. In other words, it is suggested that holes injected from $\alpha$ NPD can be transported to the cathode in Alq3 layer in spite of lower hole mobility than electron mobility[19]. If the carrier recombination occurs in Alq3 for the inverted-type device, it is expected that the recombination zone is located near the cathode. 
We prepared two partially guest-doped specimens with PEI. The guest dye is a famous 3(benzothiazo-2-yl)-7-(diethylamino)-2H-chromen2-one (C540) [20]. The other (cathode side) is ITO/PEI/ C540-dope Alq3 (10 nm, $1 \mathrm{~mol} \%) /$ Alq3 $(40 \mathrm{~nm}) / \alpha$-NPD $(50 \mathrm{~nm}) / \mathrm{MoOx}(0.8 \mathrm{~nm}) / \mathrm{Ag}$. Figure 4 shows the EL spectra of two partiallydoped specimens with PEI. One (anode side) is ITO/PEI/Alq3 (40 nm)/C540-dope Alq3 (10 nm, 1 mol\%)/ $\alpha$-NPD $(50 \mathrm{~nm}) / \mathrm{MoOx}(0.8 \mathrm{~nm}) / \mathrm{Ag}$. The PL and EL spectra of C540 are similar to the EL spectrum of the anode side specimen. The EL spectrum of the cathode side specimen agrees with that of Alq3 OLED. That is, the carrier recombination zone is located near $\alpha$-NPD for the specimen with PEI. Since the EL is not observed at all for the specimen without PEI, the location of carrier recombination cannot be clarified directly by the partial doping method. Of course, we carried out the partial doping to the specimen without PEI. From the above results, although only hole injection to Alq3 layer without electron injection will give hole transportation to Alq3, the occurrence of a small electron injection to Alq3 layer will lose the hole transportation of Alq3.

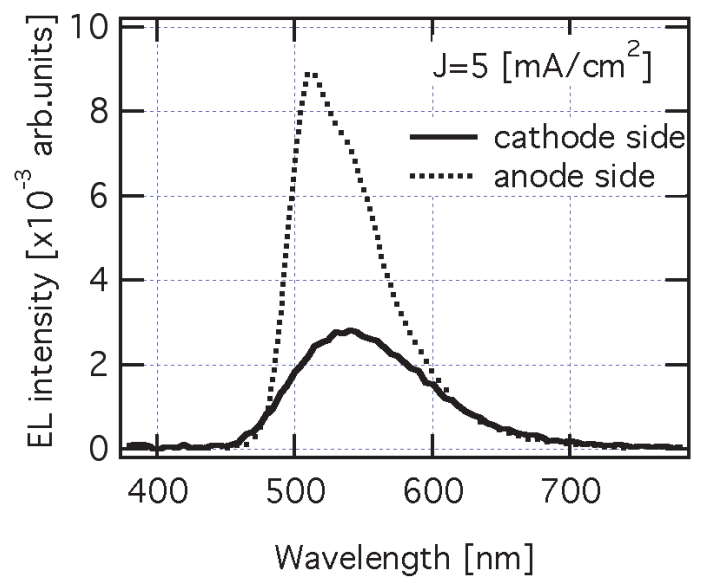

Fig. 4. The EL spectra of two partially C540-doped specimens with PEI: $10 \mathrm{~nm}$ cathode side (solid line); 10 nm anode side (dotted line).

\subsection{Comparison between conventional and inverted OLEDs}

Figures 5 show the current density - luminance - voltage characteristics of conventional and inverted specimens. The current density for the conventional specimen is $\geq 2$ orders higher than that for the inverted specimen. The former rapidly increases with increasing electron injection above 2
$\mathrm{V}$ but the latter moderately increases. The threshold voltage of electron injection from PEI/ITO to Alq3 is $\sim 4 \mathrm{~V}$ higher than that from $\mathrm{LiF} / \mathrm{Al}$ to Alq3.
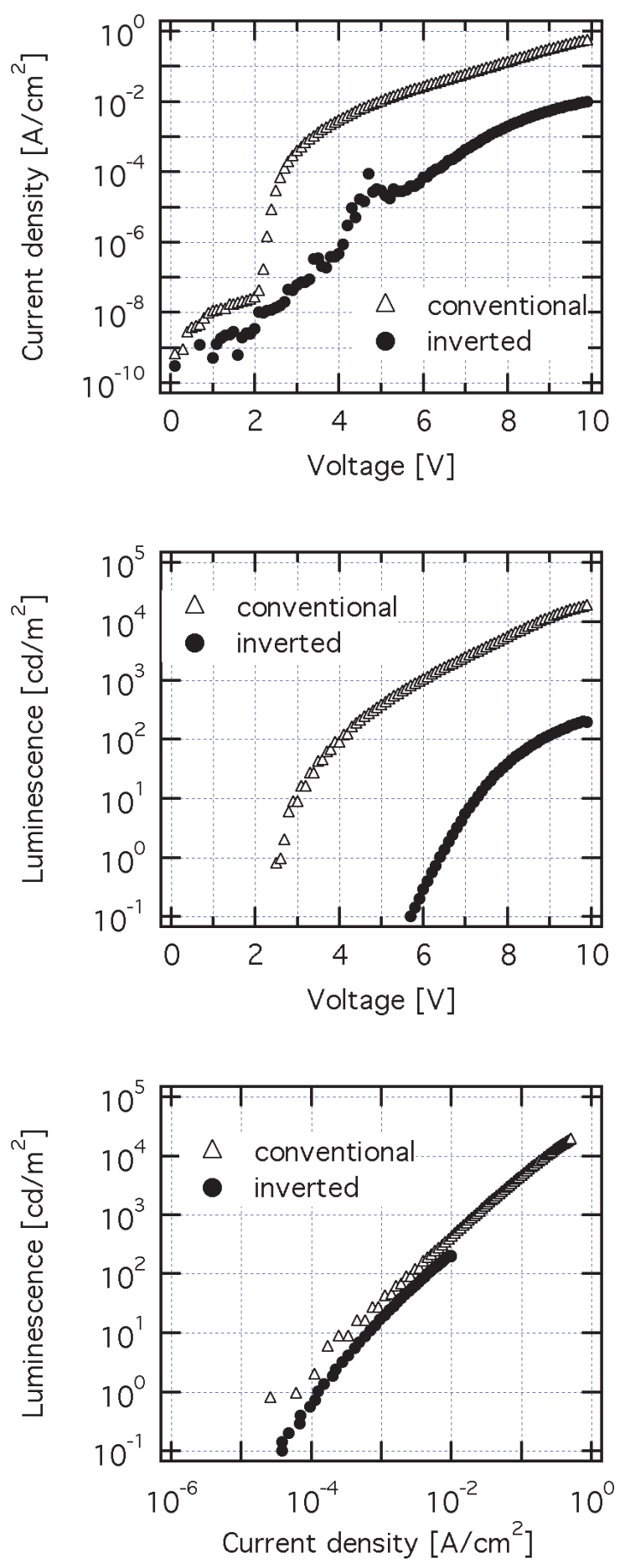

Fig. 5. The current density - voltage (top), luminance voltage (middle), and luminance - current density (bottom) characteristics for conventional and inverted OLEDs: conventional, ITO/ $\alpha$-NPD (50 nm)/Alq3 (50 $\mathrm{nm}) / \mathrm{LiF} / \mathrm{Al}$; inverted, ITO/PEI/Alq3 (50 nm)/ $\alpha-\mathrm{NPD}$ $(50 \mathrm{~nm}) / \mathrm{MoOx} / \mathrm{Ag}$. 
In this conventional type, since we do not use any hole injection material, the small barrier of hole injection from ITO to $\alpha$-NPD exists (Fig. 6). Therefore, it is thought that $\mathrm{Ag} / \mathrm{MoOx}$ anode has better potential of hole injection than ITO anode. The amount of current density depends on only the electron injection. The luminance-current density curve of the inverted OLED is not linear in the low current region.

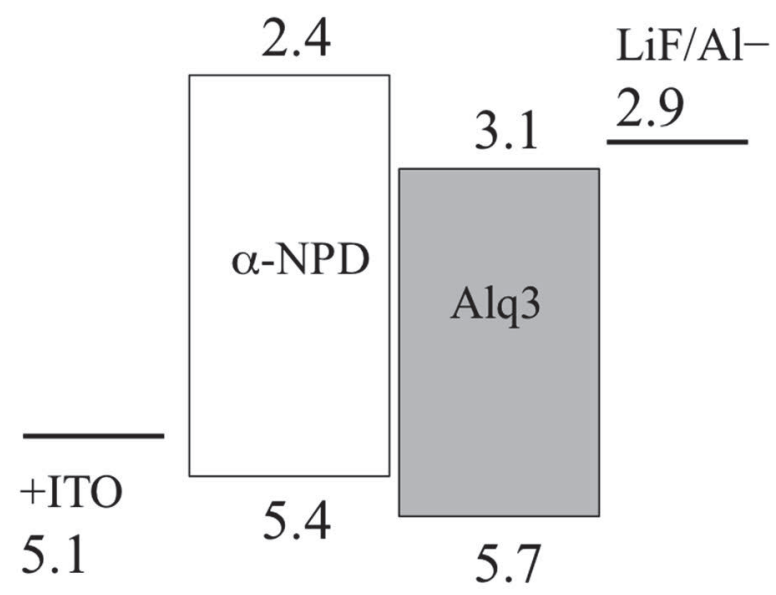

Fig. 6. The energy band of conventional structure of ITO (anode)/ $\alpha$-NPD (50 nm)/ Alq3 (50 nm)/ LiF/Al (cathode).

Figure 7 shows the EL efficiency - current density characteristics for conventional and inverted OLEDs. Although both conventional and inverted OLEDs have same hole transport and emission materials except for electrodes, the EL efficiency per current for the inverted OLED is $1 / 3-1 / 2$ poorer than that for the conventional OLED. In the conventional OLED, the luminance-current density curve in Fig. 5 looks like line but the EL efficiency shows the maximum around $0.1 \mathrm{~A} / \mathrm{cm}^{2}$ and roll-off. On the other hand, in the inverted OLED, the EL efficiency shows the maximum below $10^{-2} \mathrm{~A} / \mathrm{cm}^{2}$ and surprisingly roll-off in spite of 1-order smaller current density. In general, the roll-off phenomenon is reported to relate to the triplet-triplet annihilation accompanied with dense concentration of exciton or the imbalance of carrier balance factor, etc. [21]. Usual Alq3-based OLEDs show the maximum efficiency around $10^{-2} \mathrm{~A} / \mathrm{cm}^{2}$ because of low PL quantum efficiency [21]. In the high performance material such as phosphorescence and thermally activated delayed fluorescence (TADF) with high internal quantum efficiency, the roll-off occurs in the low current region of $10^{-4} \mathrm{~A} / \mathrm{cm}^{2}$.

However, since the roll-off threshold of our inverted OLED is lower than that of the conventional OLED but the former efficiency is poorer than the latter, low EL efficiency for the inverted OLED is not explained by exciton interaction such as exciton annihilation. The another reason for low EL efficiency of the inverted OLED is thought to be a field induced quenching. Although the driving voltage of the inverted OLED is more than double for that of the conventional OLED but the estimated electric field in Alq3 layer is same order in both OLEDs. Now we discuss the mechanism of roll-off in low performance in detail.

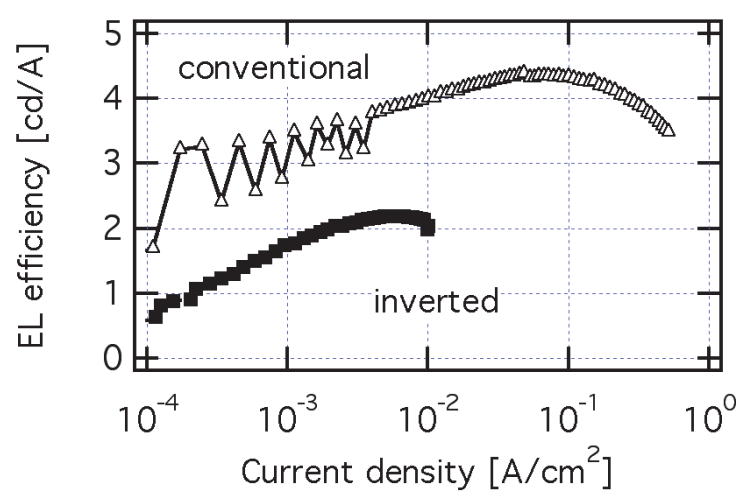

Fig. 7. The EL efficiency (luminance per current density) - current density characteristics for conventional and inverted OLEDs.

\section{Conclusion}

We fabricated the inverted OLED based on $\alpha$ NPD/Alq3 bilayer and used polyethylene -imine as an electron injection layer. When electrons were not injected in Alq3, holes could be transported in Alq3 to the cathode. But when electrons were injected in Alq3, the hole injection to Alq3 depended on the electron injection. Although the EL efficiency of the inverted OLED was lower than that of the conventional OLED, the roll-off phenomena was observed in the lower current region. The roll-off might not be related to the exciton interaction.

\section{Acknowledgements}

This research is partially obtained by the AIT Special Grant "Development of Hybrid-Power Science \& Technology for Green-Energy", and the AIT special grant for Education and Research.

\section{References}

1. https://www.oled-info.com/foldable-oleds

2. https://www.royole.com/flexpai

3. https://www.lg.com/us/ces

4. S.-G. Park and T. Mori, Luminescence, 30 (2015) 416.

5. S.-G. Park, H. Wang, and T. Mori, Jpn. J. Appl. Phys., 52 (2013) 052102. 
6. H. Heil, J. Steiger, S. Karg, M. Gastel, H. Ortner, H. von Seggern, and M. Stößel, J. Appl. Phys., 89 (2001) 420.

7. P. Tyagi, R. Srivastava, A. Kumar, S. Tuli, and M. N. Kamalasanan, Org. Electron., 14 (2013) 1391.

8. S. Barth, U. Wolf, H. Bässler, P. Müller, H. Riel, H. Vestweber, P. F. Seidler, and W. Rieß, Phys. Rev. B, 60 (1999) 8791.

9. C. W. Tang and S. A. VanSlyke, Appl. Phys. Lett., 51 (1987) 913.

10. T. Miyashita, S. Naka, H. Okada, and H. Onnagawa, Jpn. J. Appl. Phys., 44 (2005) 3682.

11. T. Miyashita, S. Naka, H. Okada, and H. Onnagawa, IDW/AD'05, 617-620 (2005).

12. K. Morii, M. Ishida, T. Takashima, T. Shimoda, Q. Wang, Md. K. Nazeeruddin, and M. Grätzel, Appl. Phys. Lett., 89 (2006) 183510.

13. H. Fukagawa, J. Photopolym. Sci. Technol., 31 (2018) 315.

14. Y. Zhou, C. Fuentes-Hernandez, J. Shim, J. Meyer, A. J. Giordano, H. Li, P. Winget, T.
Papadopouos, H. Cheun, J. Kim, M. Fenoll, A. Dinder, W. Haske, E. Najafabadi, T. M. Khan, H. Sojoudi, S. Barlow, S. Graham, J.-L. Bredas, A. Kahn, and B. Kippelen, Science, 336 (2012) 327.

15. T. Xiong, F. Wang, X. Qiao, and D. Ma, Appl. Phys. Lett., 93 (2008) 123310.

16. M. Takada, T. Kobayashi, T. Nagase, and H. Naito, Jpn. J. Appl. Phys., 55 (2016) 03DC06.

17. M. Ruscello, S. Stolz, D. L. G. Arellano, F. Ullrich, S. Hillebrant, E. Mankel, A. Pucci, W. Kowalsky, T. Emrick, A. L. Briseno, and G. Hernadez-Sosa, Org. Electron., 50 (2017) 384.

18. T. Mori, M. Imanishi, and T. Nishikawa, Appl. Phys. Exp., 4 (2011) 071601.

19. R. G. Kepler, P. M. Beeson, S. J. Jacobs, R. A. Anderson, M. B. Sinclair, V. S. Valencia, and P. A. Cahill, Appl. Phys. Lett., 66 (1995) 3618.

20. C. W. Tang, S. A. VanSlyke, and C. H. Chen, J. Appl. Phys., 65 (1989) 3610.

21. N. C. Giebink and S. R. Forrest, Phys. Rev. B, 77 (2008) 235215. 